\title{
Caracterização físico-química e dielétrica de óleos biodegradáveis para transformadores elétricos
}

\author{
Claudia R. Silva ${ }^{1}$, Maria W. N. C. Carvalho², Líbia de S. Conrado ${ }^{2}$, \\ Marcus V. L. Fook ${ }^{3} \&$ Krsthianna P. dos S. Leite ${ }^{2}$
}

\begin{abstract}
RESU M O
O óleo mineral, originário do petróleo, é o fluido isolante tradicionalmente utilizado em transformadores elétricos. Diante do apelo por fontes de energia limpa e renovável o setor elétrico também é pressionado a apresentar alternativas ao fluido de origem fóssil. Neste estudo, óleos de algodão, babaçu, girassol, milho e soja, foram avaliados quanto ao seu potencial para utilização como fluido dielétrico. As propriedades avaliadas foram densidade, viscosidade, acidez, tensão de ruptura, fator de perda, teor de água e corrosividade. O s resultados obtidos foram comparados aos limites estabelecidos na NBR 15422. Os óleos vegetais apresentaram densidade e viscosidade dentro dos limites recomendados; além disso, não se mostraram corrosivos mas devem ser submetidos a tratamentos específicos que os conduzam a atender outras especificações; o tratamento dos óleos com solução de hidróxido de sódio diminuiu a acidez, melhorou a tensão de ruptura e diminuiu o fator de perda.
\end{abstract}

Palavras-chave: óleo mineral, óleo isolante vegetal, transformador

\section{Physico-chemical and dielectric characterization of biodegradable oils for electric transformers}

\begin{abstract}
The mineral oil, originated from petroleum, is the insulating fluid traditionally used in electrical transformers. Responding to appeals for clean and renewable energy sources, the electrical sector is also under pressure to present alternatives to the fluid of fossil origin. In this study, cotton, 'babassu', sunflower, corn and soybean oils were evaluated for their potential of utilization as a dielectric fluid. The properties investigated were density, viscosity, acidity, breakdown voltage, loss factor, water content and corrosivity. The results were compared with the values of the limits established in N BR 15422. Vegetable oils show ed density and viscosity within the limits set by standard, however, higher than those presented by the studied mineral oil. It was found that vegetable oils have to pass through improvements to meet other specifications required. The treatment of oils with sodium hydroxide solution reduced the acidity, improved the breakdown voltage thereby lowering the loss factor.
\end{abstract}

Key words: mineral oil, insulating vegetable oil, transformer

${ }^{1}$ D EQ/U FSCar, Rod. W ashington Luiz, km 235, M onjolinho, CEP 13565-905, São Carlos, SP. Fone: (16) 3351-8697. E-mail: claudiacr81@hotmail.com 2 U AEQ/U FCG , Av. A prígio Veloso 882, Bodocongó, CEP 58109-970, Campina Grande, PB. Fone: (83) 2101-1115. E-mail (s): wilma@deq.ufcg.edu.br; libiac@deq.ufcg.edu.br; krsthianna_cg@hotmail.com 


\section{INTRODUÇÃO}

Transformadores são equipamentos de extrema importância nos sistemas de conversão e distribuição de energia elétrica e estão presentes desde a planta geradora, elevando a tensão para níveis adequados à transmissão a longas distâncias, até a distribuição, reduzindo a tensão para níveis de consumo residencial. Tal aplicabilidade faz dos transformadores os equipamentos mais importantes do sistema elétrico de potência.

Um componente básico do transformador é o óleo, que promove a refrigeração e o isolamento dos circuitos elétricos e magnéticos. A popularidade do óleo mineral, oriundo do petróleo se deve, nesta aplicação, à disponibilidade, ao baixo custo e às suas excelentes propriedades dielétricas e refrigerantes (Georgilakis, 2011). A partir do século 20 surgiram vários problemas de segurança ambiental (Ribeiro, 2010; Carioca et al., 2010; Mariano et al., 2008), com o desenvolvimento dos fluidos dielétricos de origem mineral (Life et al., 2010; Kwofie et al., 2011), tornando extremamente atrativo e importante o uso de produtos com alta biodegrabilidade (Meshram et al., 2011). Em decorrência da escassez do petróleo as grandes companhias mundiais do setor elétrico passaram a investigar alternativas para substituição do óleo mineral a partir de 1990 (Shogren et al., 2004; Essam \& Al-Ammar, 2010). Desde então, algumas patentes foram publicadas e os resultados de pesquisas levaram ao desenvolvimento de fluidos, isolantes vegetais comerciais. O fluido BIOTEMP foi patenteado nos Estados Unidos, em setembro de 1999, pela ABB Power T\&D Company, Inc. (Oommen \& Claiborne, 1999). Outra patente foi emitida em 1999, como resultado de estudos com óleo de soja, sob o domínio da Waverly Light \& Power (Cannon \& Honary, 1999). Em março de 2000 a patente do fluido Envirotemp FR3 foi concedida à Cooper Industries (McShane et al., 2000). Essas razões justificam o desenvolvimento de fluidos dielétricos naturais.

Ensaios realizados com fluidos dielétricos disponíveis comercialmente, demonstraram que o isolante de origem vegetal atinge ponto de combustão aos $360{ }^{\circ} \mathrm{C}$ e é $97 \%$ biodegradável em 21 dias. Destaca-se ainda que durante a combustão esses óleos só emitem dióxido de carbono e água sem derivados poliaromárticos ou silicatos prejudiciais, quando queimados. Em contraste, a queima do óleo mineral se dá aos $160^{\circ} \mathrm{C}$ e sua biodegrabilidade é de apenas $25,2 \%$ para o mesmo intervalo de tempo, levando 15 anos para ser totalmente degradado (Ferreira Junior, 2006). Além disso, o óleo vegetal é atóxico, oferece menor risco de acidentes no manuseio e armazenamento, possui melhor tolerância à umidade, tem boa rigidez dielétrica, melhor eficiência na troca térmica pelas excelentes características térmicas e ainda amplia a vida útil do equipamento. Outras vantagens relacionadas ao uso do óleo vegetal é que ele permite ampliar a potência do transformador, que passa a comportar mais kVA por quilo e ajuda a diminuir as falhas técnicas. Por outro lado, uma desvantagem dos óleos vegetais é que apresentam menor estabilidade à oxidação em comparação com óleos minerais, sendo esta característica o principal obstáculo para usá-los como base para o desenvolvimento de fluidos dielétricos (Oommen \& Claiborne, 1999). Ésteres naturais estão aumentando sua participação no mercado como fluido de isolamento de transformadores de alta potência. A característica de biodegradabilidade, alto ponto de fulgor $\left(>300{ }^{\circ} \mathrm{C}\right)$ e a possibilidade de aumentar a vida útil do papel isolante, são os pontos mais relevantes que podem ser abordados (Fofana et al., 2010). Diversos autores vêm reportando exemplos do uso de óleos vegetais como óleo isolante aplicado em transformadores de distribuição de energia, tais como óleo de palma, canola e oliva (Essam \& Al-Ammar, 2010), óleo de milho e algodão (Shah \& Tahir, 2011).

Wilhelm et al. (2009) apresentaram uma análise comparativa de propriedades térmicas, físico-químicas e elétricas de algumas variedades de óleos vegetais com relação ao óleo mineral e constataram que os óleos vegetais têm viscosidades semelhantes às do óleo de silicone e muito inferior à do fluido comercial HMWH (High Molecular Weight Hydrocarbons), usados pelo setor elétrico. Baseados em tal comprovação, os autores defenderam que óleos vegetais podem ser facilmente utilizados sem necessidade de redução da viscosidade; todavia, destacaram a possibilidade de alterar essa propriedade para níveis desejados, mediante a adição de fluidos adequados. Para diagnosticar a qualidade de novos óleos isolantes ou em serviço, são realizados ensaios estabelecidos em normas. A NBR 15422 da ABNT (2006c) serve como guia para utilização de óleo vegetal isolante para equipamentos elétricos.

Com base no exposto considera-se imprescindível caracterizar óleos vegetais disponíveis no Brasil, no sentido de se chegar a alternativas viáveis uma vez que, em regra, materiais comerciais apresentam segredos de preparação. Assim, o objetivo do trabalho é apresentar os resultados da investigação de características físico-químicas e dielétricas dos óleos de algodão, babaçu, girassol, milho e soja da classe comestível. As propriedades avaliadas foram: densidade, viscosidade cinemática, índice de acidez, corrosividade, teor de água, rigidez dielétrica e fator de perdas. O conhecimento dessas propriedades é fundamental pois são elas, dentre outras, que garantem a eficiência do sistema de isolamento e a refrigeração em transformadores (Sidibé et al., 2010; Zhang et al., 2006). Os resultados obtidos para os óleos vegetais foram comparados aos limites indicados na NBR 15422; usou-se também, como comparativo, um óleo mineral regenerado.

\section{Material e MÉTOdos}

Foram avaliadas Cinco tipos de óleo vegetal refinados da classe comestível, disponíveis no mercado brasileiro, foram avaliados, dentre os quais os óleos de algodão, babaçu, girassol, milho e soja.

Os óleos adquiridos apresentavam aspecto claro, límpido e isento de materiais em suspensão, como recomenda a NBR 15422 (ABNT 2006c). A observação do aspecto visual do óleo é item importante para fins de isolamento elétrico. A presença de materiais em suspensão, por exemplo, tem consequência direta nas características térmicas e dielétricas do fluido isolante. Cada amostra foi preservada em sua embalagem original, até o momento de uso.

O óleo mineral regenerado foi doado pela Companhia HidroElétrica do São Francisco (CHESF). Não se obtiveram 
informações a respeito das etapas pelas quais o óleo mineral foi submetido durante a regeneração; sabe-se, no entanto, que este processo é uma ação corretiva que se aplica para a retirada de água, compostos ácidos, gases dissolvidos e sedimentos do óleo envelhecido, recompondo suas características, deixando-os pronto para reutilização como fluido dielétrico.

As propriedades avaliadas para cada espécie de óleo são descritas a seguir.

A determinação da densidade dos óleos a $20^{\circ} \mathrm{C}$ foi realizada através do método da picnometria. Os experimentos foram realizados em triplicata.

A viscosidade cinemática dos óleos foi determinada com o uso do viscosímetro capilar Cannon-Fenske imerso em banho, com temperatura controlada. O ensaio de viscosidade foi realizado nas três temperaturas recomendadas pela NBR 15422 (ABNT 2006c): $20,40 \mathrm{e} 100^{\circ} \mathrm{C}$ e os experimentos foram realizados em triplicata.

A acidez dos óleos foi determinada por meio da metodologia que utiliza solução de hidróxido de sódio como titulante. $\mathrm{O}$ cálculo do índice de acidez foi realizado de acordo com a Eq. 1:

$$
\text { Índice de Acidez }=\frac{\text { a.f.M.e }}{\mathrm{p}}
$$

a - volume em $\mathrm{mL}$ da solução de hidróxido de sódio usado na titulação

f - fator de correção da solução de hidróxido de sódio (determinado por padronização da solução)

p - massa em gramas de óleo usada

e - equivalente grama do hidróxido de potássio

M - concentração molar da solução titulante

A verificação da corrosividade do óleo por presença de compostos de enxofre foi realizada pela imersão de fitas de cobre em amostras de óleo. Os frascos contendo óleo de fita foram fechados e levados à estufa a $150^{\circ} \mathrm{C}$, por $48 \mathrm{~h}$, conforme procedimento descrito na NBR 10505 (ABNT, 2006a). Passado o tempo do teste, realizou-se a avaliação da cor das fitas de cobre e se classificou o óleo como corrosivo ou não-corrosivo, de acordo com a escala de cores apresentada na norma mencionada. $\mathrm{O}$ experimento foi realizado em triplicata.

O teor de água das amostras foi verificado pelo método Karl Fischer, usando-se o Titulador Water Content Measuring Test Equipment Aquameter KFM 3000 fabricado pela Baur e de acordo com os critérios recomendados pela NBR 10710 (ABNT, 2006b).

Amostras de óleo foram submetidas a uma tensão elétrica sob condições prescritas no método de ensaio NBR 6869 (ABNT, 1989), exceto pelo fato de que o aumento de tensão foi

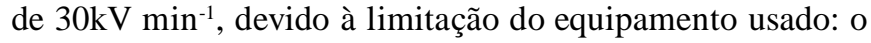
Fully Automatic Insulating Oil Tester DTA 100 E fabricado pela Baur. As normas recomendam $3 \mathrm{kV} \mathrm{min}{ }^{-1}$.

O fator de perdas dielétricas foi determinado através do Sistema Automático para a Medida do Fator de Dissipação e Resistividade DTL, fabricado pela Baur. A norma usada como referência para o ensaio foi a NBR 12133 (ABNT, 1991).
Os óleos vegetais objetos deste estudo, apresentaram acidez superior ao limite estabelecido na NBR 15422 (ABNT 2006c). Após esta constatação procedeu-se à neutralização de $1 \mathrm{~L}$ de cada amostra de óleo, através do tratamento com solução de hidróxido de sódio; após a neutralização as caracterizações físico-químicas e dielétricas foram realizadas novamente a fim de verificar se o processo de neutralização modificou as características iniciais.

\section{RESULTADOS E DISCUSSÃO}

Os índices de acidez dos óleos estão dispostos na Tabela 1. Os óleos vegetais comestíveis apresentaram acidez superior à do limite estabelecido na NBR 15422 (ABNT 2006c), de 0,06 $\mathrm{mgKOH} \mathrm{g}^{-1 ;}$ em função disto, foram realizadas neutralizações (tratamento com solução de $\mathrm{NaOH}$ ) dos óleos para deixá-los com os níveis recomendados de acidez.

Tabela 1. Índice de acidez dos óleos comerciais após tratamento de neutralização

\begin{tabular}{lcc}
\hline & \multicolumn{2}{c}{ Índice de acidez $\left(\mathrm{mg} \mathrm{KOH}^{-1}\right.$ ) } \\
\hline Tipo de óleo & Óleo comercial & Óleo comercial tratado com NaOH \\
Algodão & $0,14 \pm 0,00$ & $0,05 \pm 0,00$ \\
Babaçu & $0,27 \pm 0,01$ & $0,04 \pm 0,00$ \\
Girassol & $0,14 \pm 0,00$ & $0,04 \pm 0,00$ \\
Milho & $0,22 \pm 0,00$ & $0,03 \pm 0,00$ \\
Soja & $0,20 \pm 0,01$ & $0,03 \pm 0,00$ \\
\hline
\end{tabular}

Os resultados apresentados na Tabela 1 indicam que, após o procedimento de neutralização, todos os óleos alcançaram índice de acidez inferior ao máximo recomendado na NBR15422.

São apresentados na Tabela 2 os valores de viscosidade e densidade dos óleos vegetais após processo de neutralização. Os resultados indicam que os óleos de algodão, girassol, milho e soja, são cerca de três vezes mais viscosos que o óleo mineral; contudo, nas três temperaturas a viscosidade está dentro dos limites estipulados na NBR 15422 (ABNT, 2006c). A densidade desses óleos também é superior à do óleo mineral; entretanto, os valores não excedem o valor máximo estabelecido. Não há diferença significativa entre as densidades e viscosidades desses óleos e as dos fluidos vegetais comerciais BIOTEMP, Envirotemp FR3. Por outro lado, o óleo de babaçu é pastoso e opaco, mesmo a $25{ }^{\circ} \mathrm{C}$, devido à elevada percentagem de triaciglicerídeos saturados naturalmente presentes na sua composição.

Assim, na temperatura de ensaio de $20{ }^{\circ} \mathrm{C}$ o óleo não apresenta características fluidodinâmicas adequadas para a utilização como fluido isolante de transformadores; apesar disto, para as temperaturas mais elevadas $\left(40\right.$ e $\left.100^{\circ} \mathrm{C}\right)$ o óleo de babaçu apresenta menor viscosidade que os demais óleos vegetais testados.

Como o resfriamento dos transformadores é realizado pelo fluxo convectivo do líquido isolante, tanto a viscosidade quanto a densidade do óleo são fatores importantes do ponto de vista da transferência de calor. Assim, baixas viscosidade e densidade do fluido isolante são desejáveis para facilitar sua circulação; ao passo em que forem excessivamente elevadas, podem inibir 
Tabela 2. Densidade relativa e viscosidade cinemática dos óleos vegetais neutralizados

\begin{tabular}{|c|c|c|c|c|}
\hline \multirow{3}{*}{$\begin{array}{c}\text { Tipo de óleo } \\
\text { Limite NBR } 15442\end{array}$} & \multirow{3}{*}{$\begin{array}{c}\text { Densidade relativa* } \\
0,96 \text { máximo }\end{array}$} & \multicolumn{3}{|c|}{ Viscosidade (cSt) } \\
\hline & & $T=20^{\circ} \mathrm{C}$ & $T=40^{\circ} \mathrm{C}$ & $T=100 \stackrel{\circ}{ }$ \\
\hline & & 150 máximo & 50 máximo & 15 máximo \\
\hline Algodão & $0,9207 \pm 000$ & $66,81 \pm 0,09$ & $33,98 \pm 0,09$ & $8,04 \pm 0,00$ \\
\hline Babaçu & - & - & $27,51 \pm 0,04$ & $6,50 \pm 0,02$ \\
\hline Girassol & $0,9249 \pm 0,00$ & $70,16 \pm 0,13$ & $32,15 \pm 0,09$ & $8,14 \pm 0,00$ \\
\hline Milho & $0,9221 \pm 0,00$ & $74,95 \pm 0,13$ & $34,80 \pm 0,06$ & $8,35 \pm 0,05$ \\
\hline Soja & $0,9239 \pm 0,00$ & $67,99 \pm 0,27$ & $31,60 \pm 0,00$ & $8,27 \pm 0,02$ \\
\hline Mineral & $0,8797 \pm 0,01$ & $20,90 \pm 0,00$ & $9,35 \pm 0,01$ & $2,74 \pm 0,00$ \\
\hline
\end{tabular}

* densidade do óleo a 20 ํ C em relação à água pura a $4 \stackrel{\circ}{\mathrm{C}}$

a capacidade de dissipação de calor e levar o equipamento a sobrecargas de temperatura. Trata-se, portanto, de dois aspectos críticos para o funcionamento seguro de transformadores, especialmente em regiões de clima frio. Em função de densidade e viscosidade superior àquela apresentada pelo óleo mineral, o uso do óleo vegetal como isolante para equipamentos elétricos pode requerer mudanças no design e no sistema de bombeamento do fluido.

Na Figura 1 valores médios da viscosidade são comparados aos limites estabelecidos na NBR 15422 (ABNT, 2006c). Verificase que os óleos vegetais neutralizados $(\mathrm{N})$ ou não, apresentam viscosidade dentro do limite estabelecido. Brock et al. (2008) encontraram resultados equivalentes.

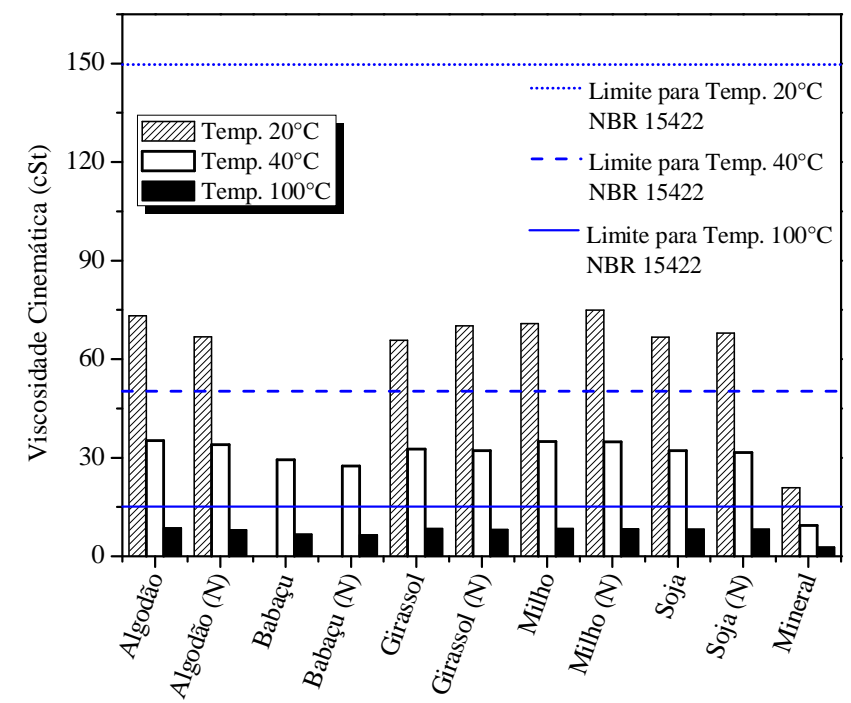

Fonte: ABNT, 2006c

Figura 1. Viscosidade dos óleos a 20,40 e $100^{\circ} \mathrm{C}$ comparada aos limites estabelecidos na NBR 15422

O resultado dos testes de corrosividade dos óleos neutralizados, apresentados na Figura 2, indica que os óleos vegetais não apresentaram comportamento corrosivo, considerando ainda que não houve mudança na coloração das fitas de cobre submetidas ao ensaio. Este comportamento não foi verificado para as fitas de cobre submetidas ao teste na presença do óleo mineral regenerado, classificado como corrosivo. De modo geral, os óleos minerais novos apresentam comportamento não corrosivo quando testados sob as condições ensaiadas de acordo com a NBR 10505 (ABNT, 2006a). Supõe-se que o óleo mineral apresentou comportamento

corrosivo por se tratar de um fluido que passou por desgaste durante seu tempo de uso.
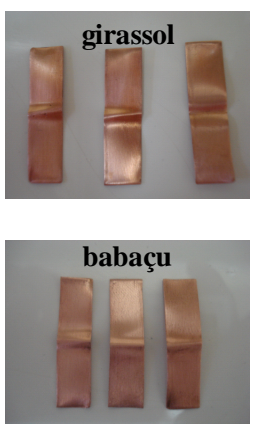
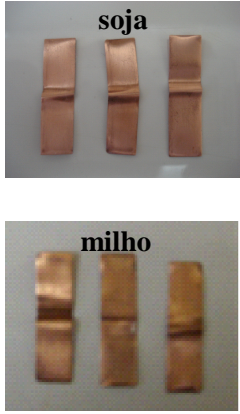
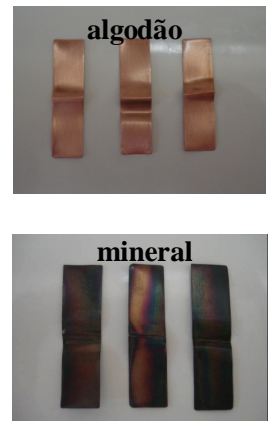

Figura 2. Fitas de cobre após ensaio de corrosividade dos óleos neutralizados

O caráter não corrosivo apresentado pelo óleo vegetal é um aspecto bastante positivo (Castelo-Branco \& Torres, 2011), haja vista que as reações de corrosão produzem sulfeto de cobre que tem elevada condutividade e, ao se dispersar sobre o papel isolante do transformador, reduz sua rigidez dielétrica.

O ensaio NBR 10505 é um teste bastante usado no setor elétrico para verificar corrosividade dos óleos. Entretanto, tratase de um teste puramente qualitativo que não deve ser considerado conclusivo para a presença ou não de compostos de enxofre. É interessante aliar este resultado em relação a outras técnicas de caracterização, para maior confiabilidade.

Fofana et al. (2010) correlacionaram o teor de água com as propriedades dielétricas dos óleos vegetais e mineral, convertendo as umidades absolutas em relativas de acordo com a Eq. 2. O cálculo da umidade relativa é importante porque óleos de origem mineral e vegetal possuem solubilidades diferentes. $\mathrm{Na}$ temperatura de $25{ }^{\circ} \mathrm{C}$ óleos de origem vegetal absorvem aproximadamente 1000 ppm de água enquanto o óleo mineral absorve entre 55 e 60 ppm (Oommen \& Claiborne, 1999). A umidade relativa do óleo $\left(\mathrm{W}_{\text {rel }}\right)$ corresponde à quantidade de água dissolvida no óleo em relação à capacidade máxima de umidade que o óleo pode suportar.

$$
\mathrm{W}_{\mathrm{rel}}=\frac{\mathrm{W}_{\mathrm{abs}}}{\mathrm{W}_{\mathrm{L}}(\mathrm{T})}
$$

onde:

$\mathrm{W}_{\mathrm{abs}}$ - teor de água absoluto do óleo

$\mathrm{W}_{\mathrm{L}}(\mathrm{T})$ - limite de saturação do óleo 
As amostras de óleos vegetais comerciais não neutralizados e após neutralização apresentaram teor de água absoluto entre $843 \pm 8$ e $961 \pm 3$ ppm; já o óleo mineral apresentou teor de água absoluto de $50 \pm 3$ ppm. Os valores de $\mathrm{W}_{\mathrm{abs}}$ são bem distintos do óleo mineral quando comparados com os de qualquer óleo vegetal avaliado no presente trabalho. Entretanto, as umidades relativas são próximas, independente do óleo ser de origem vegetal ou mineral. Os valores de $\mathrm{W}_{\text {rel }}$ dos óleos vegetais

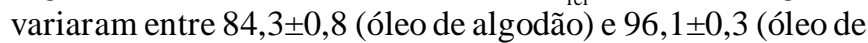

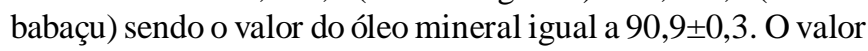
deste último praticamente é a média entre os valores de menor e maior umidade relativa dos óleos vegetais. Este fato se deve aos limites de saturação dos óleos vegetais e minerais $\mathrm{W}_{\mathrm{L}}(\mathrm{T})$, na temperatura estudada, que são semelhantes aos das umidades absolutas $\mathrm{W}_{\mathrm{abs}}, 800$ a1000 ppm para os óleos vegetais e 50 a 60 ppm para o óleo mineral. Resultados semelhantes têm sido reportados na literatura (Fofana et al., 2010).

A presença de umidade é um aspecto que merece atenção pois acelera a reação com a celulose do papel isolante, conduzindo-a à degradação. Além disso, também provoca diminuição da eficiência de impregnação do óleo no papel isolante, levando a uma diminuição do ciclo de vida do sistema de isolamento. Desta forma, o teor de água apresentado pelos óleos vegetais comestíveis é um parâmetro que precisa sef melhorado para o funcionamento seguro do equipamento que venha a utilizá-los como líquido dielétrico, independente das umidades relativas.

Realizou-se a secagem de óleo de girassol utilizando-se estufa a vácuo $\left(100 \mathrm{~mm} \mathrm{Hg}\right.$ ) a 60 e $80^{\circ} \mathrm{C}$ durante seis horas e se constatou que referidas condições não foram eficientes para remoção de umidade a um nível exigido para uso do óleo como isolante elétrico. Obteve-se, como resultado, um óleo com cerca de 700 ppm de água. Também se realizou a secagem em estufa convencional a $100{ }^{\circ} \mathrm{C}$ durante seis horas, condições induziram ao primeiro sinal de oxidação do óleo, o ranço. Concluiu-se, portanto, que é preciso investir em métodos alternativos que diminuam o teor de água sem comprometer outras propriedades do óleo como, por exemplo, processos adsortivos para retirada de água (Oliveira et al., 2010; El-Din et al., 2011).

As Figuras 3A e 3B apresentam os gráficos com os valores médios da rigidez diétrica e perda de carga dos óleos vegetais não neutralizados e neutralizados.

Observa-se, na Figura 3A, que as cinco espécies de óleos vegetais não neutralizados apresentaram rigidez dielétrica, inferior ao mínimo especificado na NBR 15422, que é $30 \mathrm{kV}$. Este fato pode estar relacionado ao elevado teor de água, para níveis de isolação elétrica. O máximo teor de água recomendado em norma é 200 ppm. Tem-se verificado que a rigidez dielétrica de óleos vegetais se mantém acima de $30 \mathrm{kV}$ para concentrações de água abaixo de 500 ppm (Lizhi et al., 2008). Nenhum dos óleos vegetais estudado neste trabalho possui teor de água igual ou menor que o recomendado pela norma.

Como já mencionado, o óleo de babaçu apresentou a maior umidade relativa dentre os óleos avaliados e, consequentemente, menor rigidez dielétrica. O óleo mineral também apresentou baixa rigidez dielétrica.

$\mathrm{Na}$ Figura 3A verifica-se que os óleos neutralizados apresentaram melhoria na rigidez dielétrica, com destaque para
A.

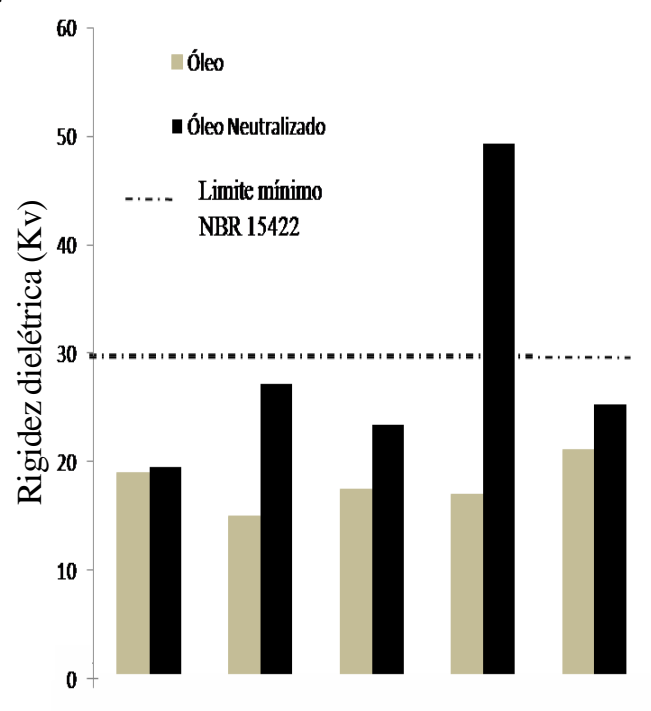

B.

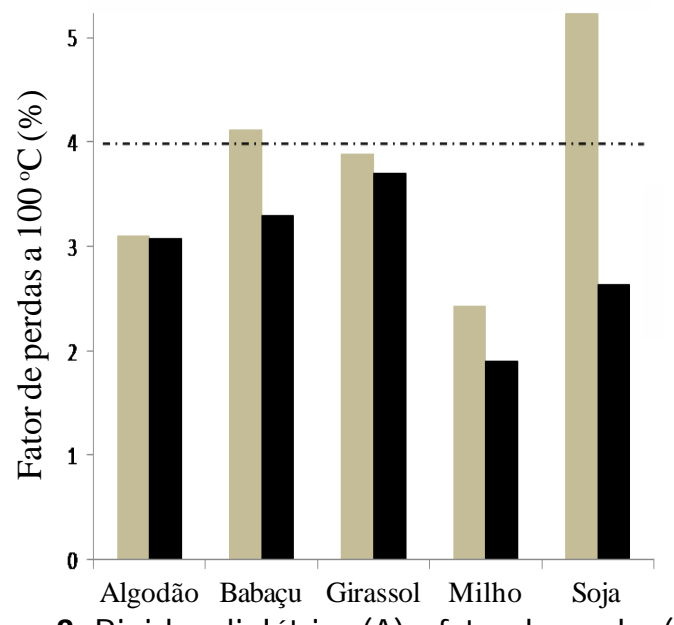

Figura 3. Rigidez dielétrica (A) e fator de perdas (B) dos óleos vegetais antese após neutralização

o óleo de milho que apresentou rigidez dielétrica superior a 40 $\mathrm{kV}$; os demais óleos não apresentaram a rigidez dielétrica mínima recomendada para fluidos dielétricos.

O fator de perdas máximo especificado na NBR 15422 (ABNT, 2006c) nas condições ensaiadas, é 4,0\%. Como visto na Figura 3B, os óleos de soja e babaçu comerciais não neutralizados não atenderam a este requisito, indicando a presença de umidade e outros produtos de deterioração do óleo.

O processo de neutralização também favoreceu a diminuição das perdas dielétricas, o que pode ser constatado na Figura 3B.

Acredita-se então, que a melhoria nas propriedades dielétricas pode estar relacionada à remoção de partículas condutoras durante a neutralização. Independentemente do teor de água, umidade relativa dos óleos neutralizados ou não neutralizados serem aproximadamente os mesmos e embora o óleo seja comestível, conduz impurezas para níveis de isolamento elétrico.

\section{ConClusõEs}

1. Os óleos de algodão, girassol, milho e soja, possuem viscosidade e densidade adequadas para uso como isolante 
em transformador. O óleo de babaçu não possui fluidodinâmica adequada a $20^{\circ} \mathrm{C}$.

2. Os óleos vegetais não apresentaram comportamento corrosivo detectável, com a aplicação da metodologia recomendada pela NBR 10505 (ABNT, 2006a).

3. Os cinco tipos de óleo vegetal não neutralizados apresentaram rigidez dielétrica inferior à especificada na NBR 15422 (ABNT, 2006c).

4. O processo de neutralização favoreceu a diminuição das perdas dielétricas e o aumento da rigidez dielétrica. Dentre os óleos neutralizados, o óleo de milho apresentou rigidez dielétrica superior ao mínimo recomendado para fluidos dielétricos.

\section{AgradeCIMENTOS}

Ao CNPq, pelo apoio financeiro.

\section{LITERATURA CITADA}

ABNT - Associação Brasileira de Normas Técnicas. NBR 6869: Determinação de rigidez dielétrica de óleos isolantes (Eletrodos de disco). Rio de Janeiro: ABNT, 1989. 13p.

ABNT - Associação Brasileira de Normas Técnicas. NBR 12133: Líquidos isolantes elétricos - Determinação do fator de perdas dielétricas e da permissividade relativa constante dielétrica). Rio de Janeiro: ABNT, 1991. 13p.

ABNT - Associação Brasileira de Normas Técnicas. NBR 10505: Óleo mineral isolante - determinação de enxofre corrosivo. Rio de Janeiro: ABNT, 2006a. 13p.

ABNT - Associação Brasileira de Normas Técnicas. NBR 10710: Determinação de água em líquidos isolantes (Método KarlFischer). Rio de Janeiro: ABNT, 2006b. 13p.

ABNT - Associação Brasileira de Normas Técnicas. NBR 15422: Óleo vegetal isolante para equipamentos elétricos. Rio de Janeiro: ABNT, 2006c. 13p.

Al-Ammar, E. A. Evaluation of seed oils based on statistical breakdown data for their application as insulating fluids in distribution transformers. European Journal of Scientific Research, v.40, p.15-26, 2010.

Brock, J.; Nogueira, M. R.; Zakrzevski, C.; Corazza, F. C.; Corazza, M. L.; Oliveira, J. V. Determinação experimental da viscosidade e condutividade térmica de óleos vegetais. Ciência e Tecnologia de Alimentos, v.28, p.564-570. 2008.

Cannon, G. S.; Honary, L.; US Patent 5.958,851, Soybean based transformer oil and transmission line fluid. 1999. 9p.

Carioca, J. O. B.; Correia, R. G.; Hiluy, J. J. F.; Macambra, S. J. Green dielectric oils; problems and perspectives, Journal of Biotechnology, v.1, p.120-128 2010.

Castelo-Branco, V. N.; Torres, A. G. Capacidade antioxidante total de óleos vegetais comestíveis: determinantes químicos e sua relação com a qualidade dos óleos. Revista de Nutrição, v.24, p.173-187, 2011.

El-Din, M. G; Hongjing, F.; Wang, N.; Ayala, P. C. Leonidas, P. E.; Przemyslaw, D.; Martin, J. W.; Zubot, W.; Smith, D. W. Naphthenic acids speciation and removal during petroleum-coke adsorption and ozonation of oil sands process-affected water. Science of the Total Environment, v.409, p.5119-5125, 2011.
Ferreira Junior, W. A CPFL cumpre seu papel, Revista P\&D: Projetos ambientais. ANEEL, v.1, p.23-24, 2006.

Fofana, I.; Hemmatjou, H. Farzaneh, M.; Low temperature and moisture effects on polarization and depolarization currents of oil paper insulation, Electric Power Systems Research, v.80, p.91-97, 2010.

Georgilakis, P. S. Environmental cost of distribution transformer losses. Applied Energy, v.88, p.3146-3155, 2011.

Kwofie, A. B.; Yeboah, P. O.; Pwamang, J. Determination of levels of polychlorinated biphenyl in transformers oil from some selected transformers in parts of the Greater Accra Region of Ghana, Chemosphere, v.82, p.103-106, 2011.

Life, K.; Hilary, I.; Inyang, J. W.; Hilger, H. Aromatic and aliphatic hydrocarbon balance in electric transformer oils. Fuel, v.89, p.3114-3118, 2010.

Lizhi, H.; Toyoda, K.; Ihara I. Dielectric properties of edible oils and fatty acids as a function of frequency, temperature, moisture and composition, Journal of Food Engineering, v.88, p.151-158, 2008.

Mariano, A. P.; Bonotto, D. M.; Angelis, D. F.; Pirôllo, M. P. S.; Contiero, J. Biodegradabilidade de óleos diesel comercial e intemperizado. Brazilian Journal of Microbiology, v.39, p.133142, 2008.

McShane, C. P.; Corkran, J. L.; Harthun, R. A.; Gauger, G. A.; Rapp, K. J.; Howells, E.; US Patent 6.037,537, Cooper Industries, Inc., Houston, TX. Vegetable oil based dielectric coolant. 2000.

Meshram, P. D.; Puri, R. G.; Patil, H. V.; Epoxidation of wild safflower (Carthamus oxyacantha) oil with peroxy acid in presence of strongly acidic cation exchange Resin IR-122 as Catalyst. International Journal of ChemTech Research. v.3, p.1152-1158, 2011.

Oliveira, J. F. G.; Lucena I. L.; Saboya, R. M. A.; Rodrigues, M. L.; Torres, A. E. B. Fernandes, F. A. N.; Cavalcante Jr., C. L.; Parente, J. E. J. S. Biodiesel production from waste coconut oil by esterification with ethanol: The effect of water removal by adsorption. Renewable Energy, v.35, p.2581-2584, 2010.

Oommen, T. V.; Claiborne C. C.; US Patent 5, 949, 017 1999. ABB Power T\&D Company Inc. (Raleigh, NC). Electrical transformers containing electrical insulation fluids comprising high oleic acid compositions.

Ribeiro, W. C. Geografia política e gestão internacional dos recursos naturais. Revista Engenharia Sanitária e Ambiental, v.24, p.69-80, 2010.

Shah, Z. H.; Tahir, Q. A. Dielectric properties of vegetable oils. Journal of Scientific Research. v.3, p.481-492, 2011.

Shogren R. L.; Petrovic, Z.; Liu, Z.; Erhan, S. Z. Biodegradation behavior of some vegetable oil-based polymersa. Journal of Polymers and the Environment, v.12, p 50-56, 2004.

Sidibé, S. S.; Blin, J.; Vaitilingom, G.; Azoumah, Y. Use of crude filtered vegetable oil as a fuel in diesel engines state of the art: Literature review Renewable and Sustainable. Energy Reviews, v.14, p.2748-2759, 2010.

Wilhelm, H. M.; Stocco, M. B. C.; Oliveira, J. de; Wilson, U. Gomes Junior, S. B. Investigacao de oleos vegetais como potenciais fluidos de seguranca. Revista Eletricidade Moderna, v.1, p.140-147, 2009.

Zhang, G.; Yongnian, N.; Churchill, J.; Kok, S. Authentication of vegetable oils on the basis of their physico-chemical properties with the aid of chemometrics. Talanta, v.70, p.293300, 2006. 